\title{
External Fixation versus Open Reduction and Internal Fixation of Pilon Fractures: A Systematic Review and Meta-analysis
}

\author{
Manaf H Younis ${ }^{1,2}$, Osama Aldahamsheh¹, Lukman Thalib², Talal Ibrahim¹ \\ 'Department of Surgery, Section of Orthopedics, Hamad Medical Corporation, ${ }^{2}$ Department of Public Health, College of Health Sciences, Qatar University, Doha, Qatar
}

\section{AbSTRACT}

Objectives: Pilon fractures are challenging to treat and associated with complications such as skin necrosis and superficial and deep infections that can potentially lead to amputation. This meta-analysis aimed to compare the postoperative outcomes following open reduction and internal fixation (ORIF) versus external fixation for pilon fractures. Methods: We searched several databases from January 1990 to July 2017 , for any observational or experimental studies that evaluated the postoperative outcomes of pilon fractures. We pooled the effect sizes using fixed-effect models that compared the postoperative outcomes of ORIF versus external fixation. Descriptive and qualitative data were also extracted. Results: Of the 485 articles identified, 13 were eligible for the meta-analysis, with a total of 683 pilon fractures in 679 patients. The pooled estimate for major infection in external fixation of pilon fractures showed comparable events compared to those who underwent ORIF (odds ratio $[\mathrm{OR}]=1.06,95 \%$ confidence interval [CI]: $0.56-1.96, I^{2}=42.2 \%$ ). External fixation was also found to be associated with higher events for minor infection $\left(\mathrm{OR}=2.83,95 \% \mathrm{CI}: 1.63 ; 4.93, I^{2}=0.00 \%\right)$, delayed union $\left(\mathrm{OR}=2.42,95 \% \mathrm{CI}: 1.02 ; 5.72, I^{2}=0.00 \%\right)$, nonunion $\left(\mathrm{OR}=1.58,95 \% \mathrm{CI}: 0.79 ; 3.18, I^{2}=0.00 \%\right)$, malunion $\left(\mathrm{OR}=3.14,95 \% \mathrm{CI}: 1.65 ; 5.97, I^{2}=0.00 \%\right)$, and posttraumatic arthritis $\left(\mathrm{OR}=2.55,95 \% \mathrm{CI}: 1.40 ; 4.63, I^{2}=0.00 \%\right)$. These results did not change even after doing sensitivity analysis comparing limited internal fixation with external fixation, uniplanar external fixation, and circular external fixator, to ORIF. Conclusions: External fixation was associated with the same chance of having adverse events that required additional procedure(s) or intravenous antibiotics compared to ORIF in pilon fractures, but the difference in bone healing complication was much more observed. ORIF allows accurate articular reduction with comparable infection rates and lower bone healing complications.

Keywords: Complications, external fixation, meta-analysis, open reduction and internal fixation, pilon fracture, systematic review

\section{INTRODUCTION}

Pilon fractures involve the dome of the distal tibia articular surface, resulting from axial loading ranging from low to high energy, and a spectrum of articular and metaphyseal injuries where the fibula may or may not be intact. These fractures account for approximately $7 \%$ of tibia fractures. ${ }^{[1]}$

The management of pilon fractures remains challenging. Significant advancements in the management of pilon fractures have taken place with the development of orthopedic surgical techniques and materials, shifting conservative treatment toward surgical intervention. However, poor outcomes, due to associated soft-tissue complications with open reduction and internal fixation (ORIF), have led to the return of less aggressive surgical management of these fractures. ${ }^{[2]}$ The importance of the soft-tissue envelope of the distal tibia and its role in both fracture healing and long-term outcomes have

\begin{tabular}{|l|l|}
\hline \multicolumn{3}{|c|}{ Access this article online } \\
\hline Quick Response Code: & Website: \\
& www.journalmsr.com \\
\cline { 2 - 2 } & \\
\end{tabular}

become better understood over the years..$^{[3-8]}$ Hence, various types of external fixators have been proposed as a definitive treatment instead of open reduction and plating to avoid further injury to the soft tissue. ${ }^{[9,10]}$

Successful treatment of pilon fractures depends on many factors including the degree of bone comminution, severity of soft-tissue injury, delay in presentation, timing of surgery, patient general condition, concomitant injuries, surgical technique, and postoperative management. ${ }^{[11,12]}$ The options for definitive surgery include either ORIF or external fixation (uniplanar, multiplanar, ring, or hybrid). ${ }^{[13,14]}$

\section{Address for correspondence: Dr. Talal Ibrahim, \\ Hamad General Hospital, P. O. Box 3050, Doha, Qatar. \\ E-mail: tibrahim5@hamad.qa}
Received : 26-11-2017
Revised : 11-12-2017
Accepted : 12-02-2018
Published Online : 07-03-2018

This is an open access journal, and articles are distributed under the terms of the Creative Commons Attribution-NonCommercial-ShareAlike 4.0 License, which allows others to remix, tweak, and build upon the work non-commercially, as long as appropriate credit is given and the new creations are licensed under the identical terms.

For reprints contact: reprints@medknow.com

How to cite this article: Younis $\mathrm{MH}$, Aldahamsheh $\mathrm{O}$, Thalib $\mathrm{L}$, Ibrahim T. External fixation versus open reduction and internal fixation of pilon fractures: A systematic review and meta-analysis. J Musculoskelet Surg Res 2018;2:41-50. 
The rate of severe complications after surgical management ranges from $10 \%$ to $55 \%$ and some of these complications can lead to amputation. ${ }^{[15-17]}$ Soft-tissue complications include wound dehiscence, superficial skin necrosis, wound infection, pin-tract infection, damage to superficial nerves, and complex regional pain syndrome, whereas bony complications include osteomyelitis, devitalization of bone fragments, nonunion, and malunion, leading to deformities. Furthermore, the quality of articular reduction and joint congruity correlates with the development of posttraumatic arthritis.

In this study, we aim to compare external fixation to ORIF for pilon fractures in adults with regard to postoperative soft-tissue complications as well as bone healing complications and posttraumatic arthritis.

\section{Materials ANd Methods}

We used the PRISMA statement criteria in reporting our meta-analysis. ${ }^{[18]}$

\section{Search strategy}

A systematic search was performed of MEDLINE using PubMed through January 1990 to July 2017 to retrieve all published studies comparing postoperative outcomes of external fixation versus ORIF in pilon fractures. The search terms were ((pilon) OR (plafond) OR (distal tibia intra articular)) AND ((external fixat*) OR (treatment) OR (fixat*) OR (ORIF)) as words in the title or abstract. The search was limited to human subjects and English language literature. Supplementary searches were performed using Embase and Cochrane library using similar search terms. Furthermore, we performed a supplementary manual search using reference lists of original research and review articles.

\section{Selection of studies}

After retrieving publications from our search, two phases of selection were carried out according to the eligibility criteria in our meta-analysis including comparative studies whether prospective or retrospective that compared external fixation in its various types to ORIF and reporting postoperative complications. Studies were excluded if they were single-arm case series, investigating other modalities of treatment or not reporting the primary outcomes (major infections).

\section{Data extraction}

Two independent authors (M. Y. and O. A.) extracted the data including where each study was conducted, year of publication, number of patients and events, demographic characteristics of the study population, methods, and details of outcomes reported. In the event of any difference of opinion, a meeting was held, and the conflict was discussed and resolved by reaching a consensus.

\section{Outcomes}

The primary outcome in our meta-analysis was major infection, which we defined as deep soft-tissue infection, osteomyelitis, or any infection that required an additional procedure (i.e., return to the operation room for debridement) and/or therapeutic intravenous antibiotic administration (other than the routine prophylactic doses). The secondary outcomes were minor infection (superficial soft-tissue infection or any infection that was managed only with a change of dressing and/or oral antibiotics), delayed union, nonunion, malunion, and posttraumatic arthritis.

\section{Quality assessment}

Quality assessment of the eligible studies was performed using the Newcastle-Ottawa scale for nonrandomized cohort studies and the Cochrane risk of bias assessment tool for randomized controlled trials (RCTs).

\section{Statistical analysis}

We performed the meta-analysis using the OpenMeta-Analyst software (Center for Clinical Evidence Synthesis, Tufts Medical Center Boston, MA, USA), using odds ratio (OR) as an effect measure, with a $95 \%$ confidence interval (CI). The analysis was performed using the fixed effect model when there was no significant heterogeneity. We performed sensitivity analyses to examine the specific associations between certain external fixation (Ex-Fix) techniques and ORIF to postoperative outcomes; (i.e. limited internal fixation with external fixation [LIFEF], uniplanar and circular externalfixators). Sensitivity and subgroup analyses were conducted by pooling the events from the studies utilizing each technique separately. Heterogeneity was assessed using $I^{2}$, where a value of $>60 \%$ was considered significant. Publication bias was explored using funnel plot and the Egger test.

\section{RESUltS}

The systematic and hand search identified 485 studies published between January 1990 and July 2017. After two phases of selection, 13 prospective and retrospective studies comparing external fixation and ORIF for the treatment of pilon fractures in adults were considered for inclusion in our meta-analysis. Figure 1 shows the process of identifying the eligible studies. Twenty-two studies were excluded because they were not comparative studies (i.e., systemic reviews or case series), nine studies investigated other treatment modalities, and two of the studies did not report any of the outcomes of interest.

\section{Characteristics of included studies}

Table 1 summarizes the characteristics of the 13 studies included in our primary analysis. The total number of patients included was 679 adults, with 683 pilon fractures, of which 330 fractures were treated using external fixation and 353 fractures treated with ORIF. One hundred and eight (108) patients from the external fixation group were treated using circular external fixators (i.e., hybrid or Ilizarov), whereas 188 patients had undergone LIFEF, and 34 patients had uniplanar external fixators alone. All the patients were adults. The average age of the patients in both groups was 42 years. The external fixation group had 121 open fractures, 
Ex-fix vs. Orif of pilon fractures: A meta-analysis
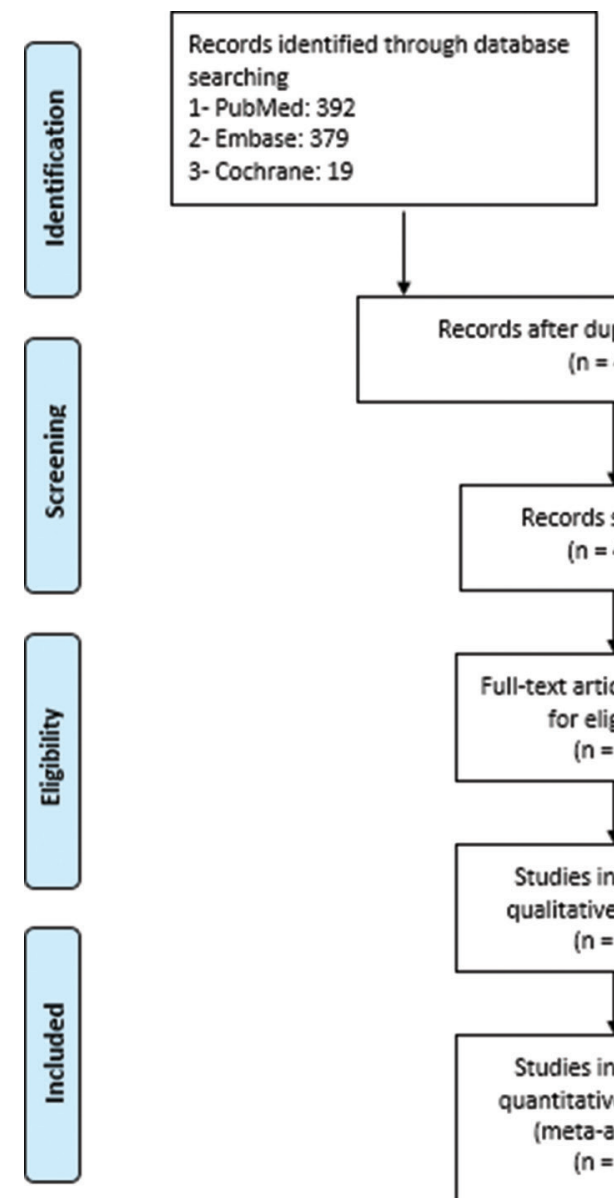

Additional records identified

through other sources

$(n=0)$
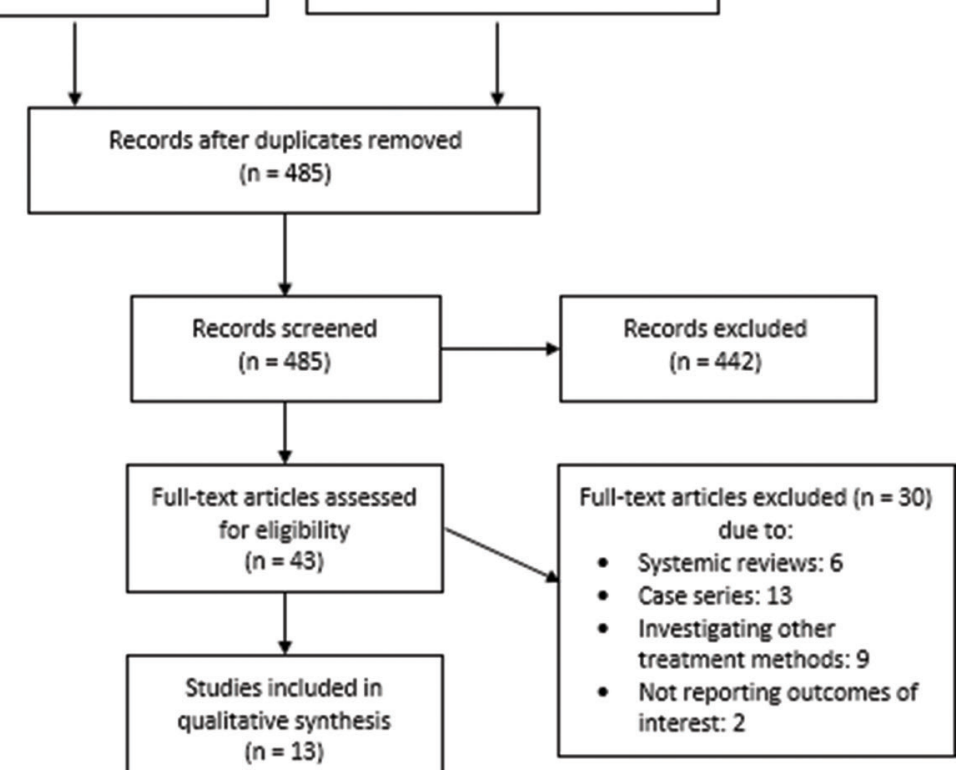

Studies included in

quantitative synthesis

(meta-analysis)

$(n=13)$

Figure 1: Flowchart of meta-analysis study selection

whereas there were 68 open fractures treated with ORIF. One study ${ }^{[26]}$ investigated only closed pilon fractures. Most of the included patients in both groups had AO/OTA Type $\mathrm{C}$ pilon fractures. However, Guo et al.$^{[29]}$ did not report the details of the type of pilon fractures included in their study. Various surgical techniques, implants, and devices were used to treat the pilon fractures in each study. This included the following uniplanar external fixation, ring external fixators, hybrid, or Ilizarov fixator with or without limited internal fixation, versus ORIF. In the majority of the cases, ORIF was performed after an average of 7-14 days, and only after the soft-tissue swelling has subsided. The average follow-up durations in the external fixation and ORIF groups were 26.2 months and 27.3 months, respectively, ranging from 5.5 months in Anglen ${ }^{[20]}$ up to 84 months in Davidovitch et al, ${ }^{[27]}$

\section{Quality assessment}

Tables 2 and 3 summarize the results for the different domains of the study quality adapted from the Newcastle-Ottawa scale for cohort studies and the Cochrane tool for assessing the risk of bias for RCTs. ${ }^{[31,32]}$ The assessment of the included cohort studies suggests that two studies ${ }^{[2,30]}$ had unbalanced prognostic factors of the patients in their cohorts as they did not match patients in the design nor did they adjust for important compounding factors, in contrast to the rest of the cohort studies. In addition, the studies of Anglen ${ }^{[20]}$ Davidovitch et al.. ${ }^{[27]}$ Guo et al. ${ }^{[29]}$ and Richards et al. ${ }^{[28]}$ did not have an adequate follow-up with $>20 \%$ of their patients lost to follow-up. The included RCTs ${ }^{[1,26]}$ carried a high risk of bias, especially in blinding and concealment of patients and outcomes. Further risk existed in the randomization process in Wyrsch et al.'s study. ${ }^{[19]}$

\section{Quantitative data synthesis}

\section{Major infections}

The comparative effect of external fixation versus ORIF in pilon fractures showed a $6 \%$ increased chance of major infection (i.e., deep soft-tissue infection, osteomyelitis, requiring a return to the operating room for a secondary procedure, and/or intravenous antibiotic administration). However, we failed to prove that this association was statistically or clinically significant $\left(\mathrm{OR}=1.06,95 \% \mathrm{CI}: 0.56,1.69, I^{2}=42.2 \%\right)$ [Figure 2]. However, when comparing LIFEF and circular Ex-Fix separately to ORIF, there was a decrease in the major infection risk, but again, these results were not statistically significant $\left(\mathrm{OR}=0.89,95 \% \mathrm{CI}: 0.37,2.17, I^{2}=53.4 \%\right.$ and $\mathrm{OR}=0.88,95 \% \mathrm{CI}: 0.20,3.95, I^{2}=55.9 \%$, respectively).

\section{Minor infections}

There was a significant trend toward increased minor 
Younis, et al.

\begin{tabular}{|c|c|c|c|c|c|c|c|c|c|c|c|c|c|c|}
\hline & Year & Country & Design & $\begin{array}{l}\text { Level of } \\
\text { evidence }\end{array}$ & $\begin{array}{l}\text { Patients } \\
(n)\end{array}$ & $\begin{array}{c}\text { Fractures } \\
\text { (n) }\end{array}$ & $\begin{array}{l}\text { Type of } \\
\text { fixation }\end{array}$ & $n$ & $\begin{array}{l}\text { Age } \\
\text { (year) }\end{array}$ & $\begin{array}{c}\text { Gender } \\
\text { male/ } \\
\text { female }(n)\end{array}$ & $\begin{array}{l}\text { Fracture } \\
\text { class }\end{array}$ & $\begin{array}{c}\text { Open } \\
(n)\end{array}$ & $\begin{array}{c}\text { Closed } \\
(n)\end{array}$ & $\begin{array}{l}\text { Follow } \\
\text { up }(m)\end{array}$ \\
\hline \multirow[t]{2}{*}{$\begin{array}{l}\text { Wyrsch } \\
\text { et al. } .^{[19]}\end{array}$} & 1996 & USA & RCT & II & 39 & 39 & EFLIF & 20 & 39 & $14 / 6$ & $\begin{array}{c}\text { I: } 4 \\
\text { II: } 4 \\
\text { III: } 12\end{array}$ & 7 & 13 & $24(25-51)$ \\
\hline & & & & & & & ORIF & 19 & 38 & $13 / 6$ & $\begin{array}{l}\text { I: } 4 \\
\text { II: } 10 \\
\text { III: } 5^{\text {b }}\end{array}$ & 3 & 16 & \\
\hline \multirow[t]{2}{*}{ Anglen ${ }^{[20]}$} & 1999 & USA & $\begin{array}{l}\text { Retrospective } \\
\text { cohort }\end{array}$ & III & 48 & 48 & $\begin{array}{l}\text { Hybrid } \\
\text { Ex-fix }\end{array}$ & 29 & 39 & $19 / 10$ & $\begin{array}{c}\text { A: } 1 \\
\text { B: } 8 \\
\text { C: } 20\end{array}$ & 8 & 21 & $20(5.5-46.9)$ \\
\hline & & & & & & & ORIF & 19 & 33 & $11 / 8$ & $\begin{array}{c}\text { A: } 3 \\
\text { B: } 5 \\
\text { C: } 11^{\text {a }}\end{array}$ & 1 & 18 & \\
\hline \multirow[t]{3}{*}{$\begin{array}{l}\text { Pugh } \\
\text { et al. } .^{[21]}\end{array}$} & 1999 & USA & $\begin{array}{l}\text { Retrospective } \\
\text { cohort }\end{array}$ & III & 60 & 60 & $\begin{array}{l}\text { Uni-planer } \\
\text { Ex-Fix }\end{array}$ & 21 & NR & NR & $\begin{array}{c}\text { A: } 2 \\
\text { B: } 2 \\
\text { C1: } 1 \\
\text { C2: } 13 \\
\text { C3:14 }\end{array}$ & 16 & 5 & NR \\
\hline & & & & & & & $\begin{array}{l}\text { Single } \\
\text { ring } \\
\text { hybrid } \\
\text { Ex-Fix }\end{array}$ & 15 & NR & NR & $\begin{array}{l}\text { A: } 4 \\
\text { B: } 1 \\
\text { C1: } 1 \\
\text { C2: } 2 \\
\text { C3: } 7\end{array}$ & 4 & 11 & NR \\
\hline & & & & & & & ORIF & 24 & NR & NR & $\begin{array}{l}\text { A: } 4 \\
\text { B: } 6 \\
\text { C1: } 6 \\
\text { C2: } 4 \\
\text { C3: } 3\end{array}$ & 6 & 18 & NR \\
\hline \multirow[t]{2}{*}{$\begin{array}{l}\text { Bocchi } \\
\text { et al. } .^{[22]}\end{array}$} & 2000 & Italy & $\begin{array}{l}\text { Retrospective } \\
\text { cohort }\end{array}$ & III & 13 & 13 & $\begin{array}{l}\text { Uniplaner } \\
\text { Ex-Fix }\end{array}$ & 9 & 52.5 & $8 / 1$ & $\begin{array}{l}\text { A: } 2 \\
\text { B: } 1 \\
\text { C1: } 2 \\
\text { C2: } 1 \\
\text { C3: } 2\end{array}$ & 5 & 4 & 12 \\
\hline & & & & & & & ORIF & 4 & 52.3 & $3 / 1$ & $\begin{array}{l}\text { A: } 1 \\
\text { B: } 2 \\
\text { C1: } 1 \\
\text { C2: } 0 \\
\text { C3: } 0\end{array}$ & 0 & 4 & 22.8 \\
\hline \multirow[t]{2}{*}{$\begin{array}{l}\text { Watson } \\
\text { et al } .^{\left[{ }^{[1}\right.}\end{array}$} & 2000 & USA & $\begin{array}{l}\text { Retrospective } \\
\text { cohort }\end{array}$ & III & 58 & 58 & ELIF & 58 & NR & NR & & 30 & 28 & $57.6(8-76.8)$ \\
\hline & & & & & 36 & 36 & ORIF & 36 & NR & NR & & 0 & 36 & \\
\hline \multirow[t]{2}{*}{$\begin{array}{l}\text { Harris } \\
\text { et al. } .^{[23]}\end{array}$} & 2006 & USA & $\begin{array}{l}\text { Retrospective } \\
\text { cohort }\end{array}$ & III & 76 & 79 & $\begin{array}{l}\text { LIF + } \\
\text { Ring } \\
\text { Ex-fix } \\
\text { (LIFEF) }\end{array}$ & 16 & 57.6 & $7 / 9$ & $\begin{array}{c}\text { A: } 0 \\
\text { B: } 1 \\
\text { C1: } 1 \\
\text { C2: } 0 \\
\text { C3: } 14\end{array}$ & 5 & 11 & $26(24-38)$ \\
\hline & & & & & & & ORIF & 63 & 40.6 & $38 / 22$ & $\begin{array}{c}\text { A: } 0 \\
\text { B: } 10 \\
\text { C1: } 14 \\
\text { C2: } 10 \\
\text { C3: } 29^{\mathrm{a}}\end{array}$ & 16 & 47 & \\
\hline \multirow[t]{2}{*}{$\begin{array}{l}\text { Koulouvaris } \\
\text { et al. } .^{[24]}\end{array}$} & 2007 & USA & $\begin{array}{l}\text { Retrospective } \\
\text { cohort }\end{array}$ & III & 55 & 55 & $\begin{array}{l}\text { Uni-planer } \\
\text { Ex-Fix }\end{array}$ & 20 & 42.0 & NR & $\begin{array}{c}\text { A: } 0 \\
\text { B: } 7 \\
\text { C1: } 1 \\
\text { C2: } 10 \\
\text { C3: } 2\end{array}$ & 9 & 11 & $77.7(38-132)$ \\
\hline & & & & & & & $\begin{array}{l}\text { Hybrid } \\
\text { Ex-Fix }\end{array}$ & 22 & 48.4 & NR & $\begin{array}{c}\text { A: } 0 \\
\text { B: } 4 \\
\text { C1: } 13 \\
\text { C2: } 1 \\
\text { C3: } 4\end{array}$ & 7 & 15 & $67.9(36-132)$ \\
\hline
\end{tabular}




\begin{tabular}{|c|c|c|c|c|c|c|c|c|c|c|c|c|c|c|}
\hline & Year & Country & Design & $\begin{array}{l}\text { Level of } \\
\text { evidence }\end{array}$ & $\begin{array}{c}\text { Patients } \\
\text { (n) }\end{array}$ & $\begin{array}{c}\text { Fractures } \\
(n)\end{array}$ & $\begin{array}{l}\text { Type of } \\
\text { fixation }\end{array}$ & $n$ & $\begin{array}{c}\text { Age } \\
\text { (year) }\end{array}$ & $\begin{array}{c}\text { Gender } \\
\text { male/ } \\
\text { female }(n)\end{array}$ & $\begin{array}{c}\text { Fracture } \\
\text { class }\end{array}$ & $\begin{array}{l}\text { Open } \\
(n)\end{array}$ & $\begin{array}{l}\text { Closed } \\
(n)\end{array}$ & $\begin{array}{l}\text { Follow } \\
\text { up }(m)\end{array}$ \\
\hline & & & & & & & $\begin{array}{l}\text { Staged } \\
\text { ORIF }\end{array}$ & 13 & 45.6 & NR & $\begin{array}{c}\text { A: } 0 \\
\text { B: } 8 \\
\text { C1: } 0 \\
\text { C2: } 5 \\
\text { C3: } 0^{\text {a }}\end{array}$ & 8 & 5 & $78.6(55-132)$ \\
\hline \multirow[t]{2}{*}{$\begin{array}{l}\text { Bacon } \\
\text { et al. }{ }^{[25]}\end{array}$} & 2008 & USA & $\begin{array}{l}\text { Retrospective } \\
\text { cohort }\end{array}$ & III & 38 & 38 & $\begin{array}{l}\text { Ilizarov } \\
\text { fixation }\end{array}$ & 13 & 32.3 & $11 / 2$ & $\begin{array}{l}\text { A: } 0 \\
\text { B: } 0 \\
\text { C1: } 1 \\
\text { C2: } 3 \\
\text { C3: } 9\end{array}$ & 3 & 10 & 12 \\
\hline & & & & & & & ORIF & 25 & 39.4 & $20 / 5$ & $\begin{array}{c}\text { A: } 0 \\
\text { B: } 0 \\
\text { C1: } 3 \\
\text { C2: } 7 \\
\text { C3: } 15^{\text {a }}\end{array}$ & 3 & 22 & \\
\hline \multirow[t]{2}{*}{$\begin{array}{l}\text { Wang } \\
\text { et al. }^{[26]}\end{array}$} & 2010 & China & $\mathrm{RCT}$ & I & 56 & 56 & LIFEF & 29 & 37.2 & $26 / 3$ & $\begin{array}{c}\text { A: } 0 \\
\text { B: } 2 \\
\text { C1: } 7 \\
\text { C2: } 13 \\
\text { C3: } 7\end{array}$ & 0 & 29 & 24 \\
\hline & & & & & & & $\begin{array}{l}\text { Staged } \\
\text { ORIF }\end{array}$ & 27 & 40.1 & $25 / 2$ & $\begin{array}{l}\text { A: } 0 \\
\text { B: } 3 \\
\text { C1: } 9 \\
\text { C2: } 10 \\
\text { C3: } 5^{\text {a }}\end{array}$ & 0 & 27 & \\
\hline \multirow[t]{2}{*}{$\begin{array}{l}\text { Davidovitch } \\
\text { et al. }{ }^{[27]}\end{array}$} & 2011 & USA & $\begin{array}{l}\text { Retrospective } \\
\text { cohort }\end{array}$ & III & 46 & 47 & LIFEF & 21 & 43 & $12 / 8$ & $\begin{array}{c}\text { A: } 0 \\
\text { B: } 0 \\
\text { C1: } 2 \\
\text { C2: } 6 \\
\text { C3: } 13\end{array}$ & 4 & 17 & $18(6-52)$ \\
\hline & & & & & & & ORIF & 26 & 39 & $17 / 9$ & $\begin{array}{c}\text { A: } 0 \\
\text { B: } 0 \\
\text { C1: } 3 \\
\text { C2: } 4 \\
\text { C3: } 19^{\mathrm{a}}\end{array}$ & 5 & 21 & $22(6-84)$ \\
\hline \multirow[t]{2}{*}{$\begin{array}{l}\text { Richards } \\
\text { et al. }^{[28]}\end{array}$} & 2012 & USA & $\begin{array}{l}\text { Prospective } \\
\text { cohort }\end{array}$ & II & 45 & 45 & LIFEF & 18 & 40.6 & NR & $\begin{array}{c}\text { A: } 0 \\
\text { B: } 0 \\
\text { C1: } 1 \\
\text { C2: } 1 \\
\text { C3: } 16\end{array}$ & 5 & 13 & 12 \\
\hline & & & & & & & ORIF & 27 & 46.9 & NR & $\begin{array}{c}\text { A: } 0 \\
\text { B: } 0 \\
\text { C1: } 1 \\
\text { C2: } 5 \\
\text { C3: } 21^{\text {a }}\end{array}$ & 8 & 19 & \\
\hline \multirow[t]{2}{*}{ Guo et al. ${ }^{[29]}$} & 2015 & China & $\begin{array}{l}\text { Retrospective } \\
\text { cohort }\end{array}$ & III & 78 & 78 & LIFEF & 26 & 41.2 & $18 / 8$ & NR & 9 & 17 & $14.2(12-21)$ \\
\hline & & & & & & & ORIF & 52 & 40.7 & $38 / 14$ & NR & 15 & 37 & 15.7 \\
\hline \multirow[t]{2}{*}{$\begin{array}{l}\text { Cisneros } \\
\text { et al. }{ }^{[30]}\end{array}$} & 2016 & Spain & $\begin{array}{l}\text { Retrospective } \\
\text { cohort }\end{array}$ & III & 31 & 31 & $\begin{array}{l}\text { Hybrid } \\
\text { Ex-fix }\end{array}$ & 13 & 43.3 & $7 / 6$ & $\begin{array}{l}\text { A: } 2 \\
\text { B: } 1 \\
\text { C1: } 1 \\
\text { C2: } 3 \\
\text { C3: } 6\end{array}$ & 9 & 4 & $24(24-70)$ \\
\hline & & & & & & & $\begin{array}{l}\text { Staged } \\
\text { ORIF }\end{array}$ & 18 & 52.6 & $7 / 11$ & $\begin{array}{l}\text { A: } 9 \\
\text { B: } 3 \\
\text { C1: } 0 \\
\text { C2: } 3 \\
\text { C } 3: 3^{\text {a }}\end{array}$ & 3 & 15 & \\
\hline
\end{tabular}

${ }^{a}$ Orthopedic Trauma Association classification. Marsh JL, Slongo TF, Agel J, Broderick JS, Creevey W, DeCoster TA, et al. Fracture and dislocation classification compendium -2007: Orthopaedic Trauma Association Classification, Database and Outcomes Committee. J Orthop Trauma 2007;21 10 Suppl: S1-133, ${ }^{b}$ Ruedi and Allgower classification. Ruedi TP, Allgower M. The operative treatment of intra-articular fractures of the lower end of the tibia. Clin Orthop Relat Res 1979;138:105-10. EFLIF: External fixation and limited internal fixation, LIFEF; Limited internal fixation with external fixation, Ex-Fix: External fixation, ORIF: Open reduction and internal fixation, RCT: Randomized controlled study, USA: United States of America, NR: Not reported 
Younis, et al.

\begin{tabular}{|c|c|c|c|c|c|c|c|c|c|c|c|c|}
\hline Domain & Item & Anglen ${ }^{[20]}$ & $\begin{array}{l}\text { Bacon } \\
\text { et al. } .^{[25]}\end{array}$ & $\begin{array}{l}\text { Pugh } \\
\text { et al. }\end{array}$ & $\begin{array}{l}\text { Bocchi } \\
\text { et al. }\end{array}$ & $\begin{array}{l}\text { Watson } \\
\text { et al. }\end{array}$ & $\begin{array}{l}\text { Cisneros } \\
\text { et al } .^{[30]}\end{array}$ & $\begin{array}{l}\text { Davidovitch } \\
\text { et al. } .^{[27]}\end{array}$ & $\begin{array}{c}\text { Guo } \\
\text { et al. }{ }^{[29]}\end{array}$ & $\begin{array}{l}\text { Harris } \\
\text { et al. } .^{[23]}\end{array}$ & $\begin{array}{c}\text { Koulouvaris } \\
\text { et al. } .^{[24]}\end{array}$ & $\begin{array}{l}\text { Richards } \\
\text { et al. } .^{[28]}\end{array}$ \\
\hline \multirow{4}{*}{$\begin{array}{l}\begin{array}{l}\text { Selection } \\
\text { (maximum of } \\
4 \text { stars) }\end{array} \\
\end{array}$} & $\begin{array}{l}\text { Representativeness } \\
\text { of the exposed cohort }\end{array}$ & $*$ & $*$ & $*$ & $*$ & $*$ & $*$ & $*$ & $*$ & $*$ & * & $*$ \\
\hline & $\begin{array}{l}\text { Selection of the } \\
\text { non-exposed cohort }\end{array}$ & $*$ & $*$ & $*$ & * & $*$ & $*$ & $*$ & $*$ & $*$ & $*$ & $*$ \\
\hline & $\begin{array}{l}\text { Ascertainment of } \\
\text { exposure }\end{array}$ & $*$ & * & $*$ & $*$ & $*$ & $*$ & $*$ & $*$ & $*$ & $*$ & $*$ \\
\hline & $\begin{array}{l}\text { Demonstration that } \\
\text { outcome of interest } \\
\text { was not present at } \\
\text { start of study }\end{array}$ & $*$ & $*$ & $*$ & $*$ & $*$ & $*$ & $*$ & $*$ & $*$ & $*$ & $*$ \\
\hline $\begin{array}{l}\text { Comparability } \\
\text { (maximum of } \\
2 \text { stars) }\end{array}$ & $\begin{array}{l}\text { Comparability of } \\
\text { cohorts on the basis } \\
\text { of the design or } \\
\text { analysis }\end{array}$ & Nil & $*$ & Nil & Nil & Nil & Nil & $*$ & $*$ & $*$ & $*$ & $*$ \\
\hline \multirow{3}{*}{$\begin{array}{l}\text { Outcomes } \\
\text { (maximum of } \\
3 \text { stars) }\end{array}$} & $\begin{array}{l}\text { Assessment of } \\
\text { outcome }\end{array}$ & * & * & $*$ & * & * & * & $*$ & $*$ & $*$ & $*$ & * \\
\hline & $\begin{array}{l}\text { Was follow-up long } \\
\text { enough for outcomes } \\
\text { to occur }\end{array}$ & $*$ & $*$ & Nil & $*$ & $*$ & $*$ & $*$ & $*$ & $*$ & $*$ & $*$ \\
\hline & $\begin{array}{l}\text { Adequacy of follow } \\
\text { up of cohorts }\end{array}$ & Nil & $*$ & Nil & $*$ & $*$ & $*$ & Nil & Nil & $*$ & $*$ & Nil \\
\hline
\end{tabular}

\begin{tabular}{lll}
\hline $\begin{array}{l}\text { Table 3: Assessing risk of bias: Randomized controlled } \\
\text { trials }\end{array}$ & Wyrsch \\
\hline Item & et al. ${ }^{[19]}$ & $\begin{array}{l}\text { Wang } \\
\text { et al. }{ }^{[26]}\end{array}$ \\
\hline Random sequence generation & High risk & Low risk \\
Allocation concealment & High risk & High risk \\
Blinding of participants and personnel & High risk & High risk \\
Blinding of outcome assessment & High risk & Unclear \\
Incomplete outcome data & Unclear & Low risk \\
Selective reporting & Low risk & Low risk \\
Other sources of bias* & High risk & Unclear \\
Risk of bias & High & High \\
\hline
\end{tabular}

*Other sources of bias: Important concerns about surgical randomization in Wyrsch et al. exists

infections when treating pilon fractures with external fixators (i.e., pin-tract or wound infections requiring only a change of dressing or oral antibiotics $)(\mathrm{OR}=2.83,95 \% \mathrm{CI}: 1.63,4.93$, $I^{2}=0.00 \%$ ) [Figure 3]. This association was reinforced more when performing subgroup analysis for LEFIF and circular Ex-Fix separately.

\section{Bone healing complications (delayed union, nonunion, and malunion)}

Furthermore, bone healing complications were more associated with external fixation; delayed union $\left(\mathrm{OR}=2.41,95 \% \mathrm{CI}: 1.02,5.72, I^{2}=0.00 \%\right)$ and malunion $\left(\mathrm{OR}=3.14,95 \% \mathrm{CI}: 1.65,5.97, I^{2}=0.00 \%\right)$ occurred more frequently in the external fixation group and were associated with long-term bone healing complications, however, nonunion risk was not significantly different among the two groups $\left(\mathrm{OR}=1.58,95 \%\right.$ CI: 0.79, 3.18, $\left.I^{2}=0.00 \%\right)$ [Figure 4]. LIFEF kept the same trend of increased bone healing complications, whereas the odds of delayed union and nonunion after circular Ex-Fix did not show a significant difference from ORIF [Table 4].

\section{Posttraumatic arthritis}

Posttraumatic arthritis was 2.6 times more frequent in the external fixation patients than those who underwent ORIF $\left(\mathrm{OR}=2.55,95 \% \mathrm{CI}: 1.40,4.63, I^{2}=0.00 \%\right)$.

Publication bias was assessed using funnel plots and Egger tests, which did not show patterns of significant bias in terms of any measured outcomes.

\section{Discussion}

This meta-analysis shows that, in patients with pilon fractures, early postoperative major complications and infections were not significantly affected by the method of definitive surgical management whether external fixation or ORIF was undertaken. However, with regard to minor infectious complications, these were 2.8 times more associated with external fixation, and this can be simply explained by the frequent superficial pin site infections associated with these devices. ${ }^{[33]}$ The utilization of external fixation to treat pilon fractures carried more than double the chance for delayed union and almost triple chance of malunion, but no significant difference in nonunion risk compared to the ORIF group. Long-term complications showed a high rate of posttraumatic ankle arthritis following different types of external fixation due to the lack of accurate articular reduction and stable rigid fixation better provided by the ORIF technique. ${ }^{[34]}$ These results were evident in any form of external fixation technique used and whether uniplanar, circular (hybrid/ Illizarov), or LIFEF frames were utilized. 
Ex-fix vs. Orif of pilon fractures: A meta-analysis

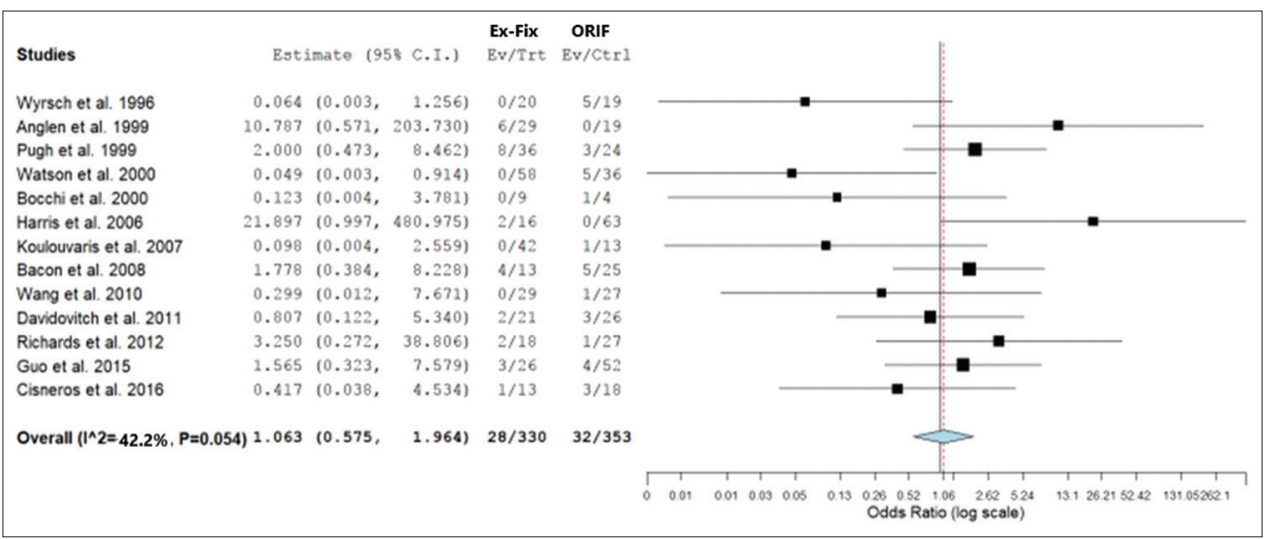

Figure 2: Meta-analysis of major infections

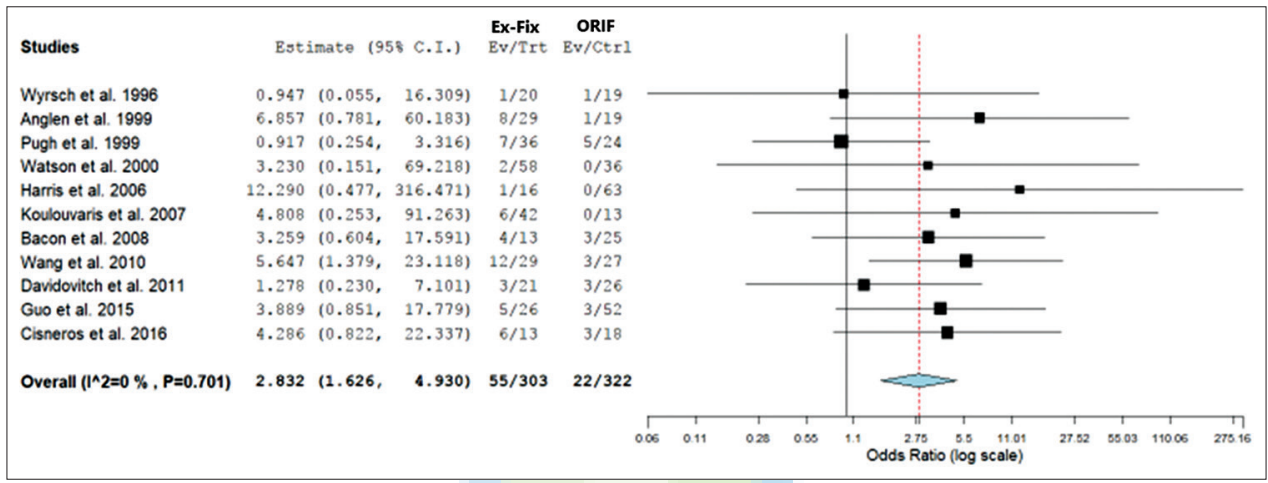

Figure 3: Meta-analysis of minor infections

Table 4: Subgroup analysis of the outcomes with different external fixation techniques

\begin{tabular}{lcccccc}
\hline & Major infection & Minor infection & Delayed union & Nonunion & Malunion & Arthritis \\
\hline Ex-Fix versus ORIF & $1.06(0.58-1.69)$ & $2.83(1.63-4.93)$ & $2.41(1.02-5.72)$ & $1.58(0.79-3.18)$ & $3.14(1.65-5.97)$ & $2.55(1.40-4.63)$ \\
LEFIF versus ORIF & $0.89(0.37-2.17)$ & $3.27(1.49-7.22)$ & $2.71(1.02-7.20)$ & $1.10(0.40-3.01)$ & $2.80(1.20-6.56)$ & $2.65(1.40-5.01)$ \\
Uniplanar Ex-Fix versus ORIF & $0.88(0.32-2.46)$ & $2.63(1.31-5.30)$ & $2.72(1.10-6.74)$ & $1.94(0.71-5.32)$ & $3.47(1.58-7.62)$ & $2.19(1.09-4.37)$ \\
Circular Ex-Fix versus ORIF & $0.88(0.19-3.95)$ & $3.03(1.31-6.99)$ & $1.16(0.12-11.51)$ & $1.37(0.55-3.38)$ & $2.70(1.07-6.83)$ & NA \\
\hline
\end{tabular}

ORIF: Open reduction and internal fixation, LIFEF; Limited internal fixation with external fixation, Ex-Fix: External fixation

There have been important advances in the surgical management and strategies of pilon fractures including hybrid external fixators, minimally invasive and limited internal fixations, staged ORIF, and arthroscopy-assisted procedures, all of which have attempted to improve the early and late outcomes of surgical treatment of such fractures. As the surgical methods continue to evolve and outcomes improve, it remains increasingly difficult to answer the ultimate question "what is the best surgical procedure to manage pilon fractures?"

The recent studies comparing external fixation and ORIF have been underpowered for major morbidities, making the results difficult to interpret. This is partially because pilon fractures are relatively uncommon, ${ }^{[5]}$ and in the present time, major infections are decreasing in rate. ${ }^{[35,36]}$ Our meta-analysis was driven mainly by the doubtful outcome favoring external fixation of pilon fractures over ORIF, which has been justifiable by many orthopedic trauma surgeons and patients wishing to avoid soft-tissue complications associated with ORIF. ${ }^{[9,19,37,38]}$ Several studies have reported higher rates of bone healing complications with external fixation. ${ }^{[19,24,25]}$ To overcome the limitations of the underpowered studies, we performed this meta-analysis pooling data from multiple studies including a total of $>670$ patients. Our meta-analysis demonstrates that short-term major morbidity is not significantly reduced by external fixation. It is notable that the results of the clinical studies included in this meta-analysis were homogeneous for all of the outcomes studied (i.e., $I^{2}<60 \%$ ); therefore, a fixed effect model has been used to analyze the data.

In our meta-analysis, it was not feasible to combine all bone healing complications in one composite outcome, as this would be considered a statistical pitfall because individual patient data were not available. To overcome the variability 
Younis, et al.

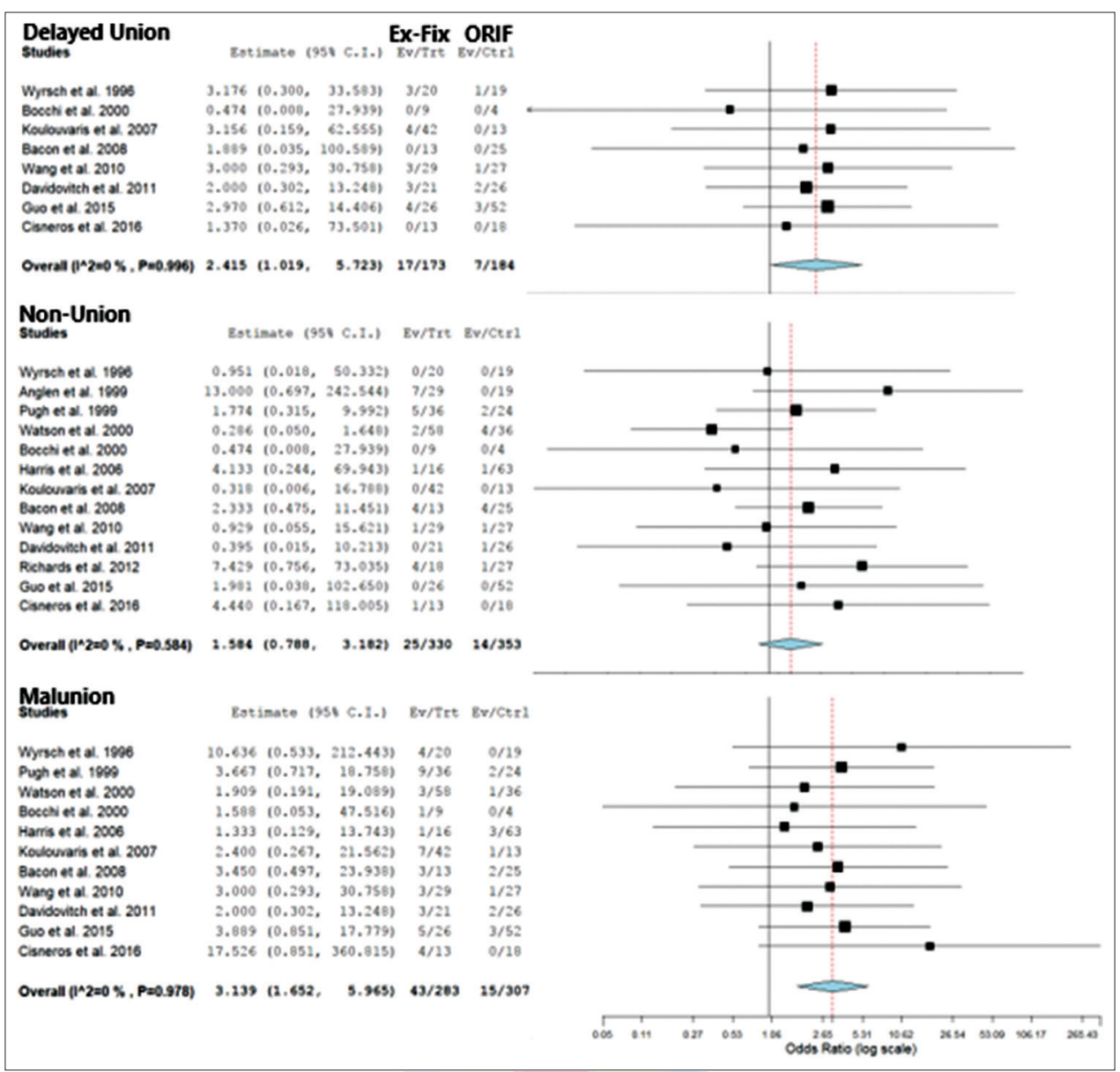

Figure 4: Meta-analysis of bone healing complications (delayed union, nonunion, and malunion)

in the several external fixation techniques used to treat pilon fractures, we have performed multiple comparisons through sensitivity analysis to examine if the usage of limited internal fixation in association with Ex-Fix or circular Ex-Fix had a different association with postoperative outcomes.

Our results must be interpreted in the light of several limitations in the current literature and included studies, consideration of the conduct and interpretation of the results of the analyses presented, and the implications of preferring one treatment over the other. The ability to detect a small scale of benefit and associated possibility of Type II error is further confounded by the relatively small sample size. Despite the reasonable homogeneity of the studies in the specific treatments being compared, there is variability in the chosen inclusion criteria, frequency and length of follow-up, and the measured outcomes in the included studies. This is a further drawback, which affects the credibility of subsequent conclusions.

Only a few prospective studies have examined the relationship between the mode of fixation and postoperative outcomes of pilon fractures. Two RCTs ${ }^{[19,26]}$ were included in our meta-analysis. The small number of randomized prospective studies forms a big challenge to the conduction of high-quality meta-analyses in surgical fields generally and in orthopedic surgery specifically. ${ }^{[39]}$ This was a study-level meta-analysis because we did not have access to individual-level data, and this has prevented us from performing subgroup analyses to see if the superiority of one fixation method over the other is affected by factors such as open/closed fractures, fracture classification, or timing to definitive fixation. Hence, important confounders could not be controlled for in this study. It should be clear when handling the results of this meta-analysis that fracture classification (Type B vs. C), degree of fracture comminution (Subtype C1 vs. C3), extent of articular involvement, and soft-tissue condition (i.e., open vs. closed fracture and soft-tissue swelling) are major determinants of surgical outcome.

Our results are consistent with the literature in that major infection risk for pilon fractures is almost the same regardless whether treated definitely with external fixation or ORIF. However, bone healing complications and posttraumatic arthritis are three times more when external fixation is used as a definitive procedure, the result that previous meta-analysis has failed to demonstrate. ${ }^{[1,34,40,41]}$ None of the previously conducted meta-analyses have looked at the comparison between external fixation and ORIF in the treatment of such complex fractures in this comprehensive way. 
Ex-fix vs. Orif of pilon fractures: A meta-analysis

\section{Conclusions}

The best surgical modality to treat pilon fractures is still equivocal and yet to be determined. While external fixators are used to treat pilon fractures by many surgeons to avoid major complications, it has been associated with high rates of delayed union, nonunion, malunion, and osteoarthritis. Therefore, we recommend ORIF of pilon fractures, when the soft-tissue condition is suitable, to obtain accurate anatomical joint reconstruction, rigid fixation of the articular surface, and restoration of distal tibia alignment. Concerns of soft-tissue complications can be addressed by meticulous preoperative evaluation and planning, whether with an early or staged protocol of ORIF.

\section{Financial support and sponsorship}

Nil.

\section{Conflicts of interest}

There are no conflicts of interest.

\section{Authors contributions}

TI conceived and designed the study. MHY \& OA conducted search, provided search materials, collected and organized data. LT \& MHY analyzed and interpreted data. MHY wrote initial and final draft of the article and provided logistic support. All authors have critically reviewed and approved the final draft and are responsible for the content and similarity index of the manuscript.

\section{REFERENCES}

1. Topliss CJ, Jackson M, Atkins RM. Anatomy of pilon fractures of the distal tibia. J Bone Joint Surg Br 2005;87:692-7.

2. Calori GM, Tagliabue L, Mazza E, de Bellis U, Pierannunzii L, Marelli BM, et al. Tibial pilon fractures: Which method of treatment? Injury 2010;41:1183-90.

3. Brumback RJ, McGarvey WC. Fractures of the tibial plafond. Evolving treatment concepts for the pilon fracture. Orthop Clin North Am 1995;26:273-85

4. Borens O, Kloen P, Richmond J, Roederer G, Levine DS, Helfet DL, et al. Minimally invasive treatment of pilon fractures with a low profile plate: Preliminary results in 17 cases. Arch Orthop Trauma Surg 2009;129:649-59.

5. Mauffrey C, Vasario G, Battiston B, Lewis C, Beazley J, Seligson D, et al. Tibial pilon fractures: A review of incidence, diagnosis, treatment, and complications. Acta Orthop Belg 2011;77:432-40.

6. Teeny SM, Wiss DA. Open reduction and internal fixation of tibial plafond fractures. Variables contributing to poor results and complications. Clin Orthop Relat Res 1993;292:108-17.

7. Sirkin M, Sanders R, DiPasquale T, Herscovici D Jr. A staged protocol for soft tissue management in the treatment of complex pilon fractures. J Orthop Trauma 1999;13:78-84.

8. Watson JT, Moed BR, Karges DE, Cramer KE. Pilon fractures. Treatment protocol based on severity of soft tissue injury. Clin Orthop Relat Res 2000;375:78-90.

9. Bone L, Stegemann P, McNamara K, Seibel R. External fixation of severely comminuted and open tibial pilon fractures. Clin Orthop Relat Res 1993;292:101-7.

10. Helfet DL, Koval K, Pappas J, Sanders RW, DiPasquale T. Intraarticular "pilon" fracture of the tibia. Clin Orthop Relat Res 1994;298:221-8.

11. Liporace FA, Yoon RS. Decisions and staging leading to definitive open management of pilon fractures: Where have we come from and where are we now? J Orthop Trauma 2012;26:488-98.
12. Mandi DM, Belin RP, Banks J, Barrett B. Pilon fractures. Clin Podiatr Med Surg 2012;29:243-78, viii.

13. Femino JE, Vaseenon T. The direct lateral approach to the distal tibia and fibula: A single incision technique for distal tibial and pilon fractures. Iowa Orthop J 2009;29:143-8.

14. Niikura T, Miwa M, Sakai Y, Lee SY, Oe K, Iwakura T, et al. Ankle arthrodesis using antegrade intramedullary nail for salvage of nonreconstructable tibial pilon fractures. Orthopedics 2009;32. pii: orthosupersite.com/view.asp?rID=41937.

15. Sands A, Grujic L, Byck DC, Agel J, Benirschke S, Swiontkowski MF, et al. Clinical and functional outcomes of internal fixation of displaced pilon fractures. Clin Orthop Relat Res 1998;347:131-7.

16. Thordarson DB. Complications after treatment of tibial pilon fractures: Prevention and management strategies. J Am Acad Orthop Surg 2000;8:253-65.

17. Dillin L, Slabaugh P. Delayed wound healing, infection, and nonunion following open reduction and internal fixation of tibial plafond fractures. J Trauma 1986;26:1116-9.

18. Moher D, Liberati A, Tetzlaff J, Altman DG, the PRISMA Group. Preferred reporting items for systematic reviews and meta-analyses: The PRISMA Statement. J Clin Epidemiol 2009;62:1006-12.

19. Wyrsch B, McFerran MA, McAndrew M, Limbird TJ, Harper MC, Johnson $\mathrm{KD}$, et al. Operative treatment of fractures of the tibial plafond. A randomized, prospective study. J Bone Joint Surg Am 1996;78:1646-57.

20. Anglen JO. Early outcome of hybrid external fixation for fracture of the distal tibia. J Orthop Trauma 1999;13:92-7.

21. Pugh KJ, Wolinsky PR, McAndrew MP, Johnson KD. Tibial pilon fractures: A comparison of treatment methods. J Trauma 1999;47:937-41.

22. Bocchi L, Maniscalco P, Bertone C, Rivera F, Crainz E. Fractures of the tibial plafond: A comparison of treatment methods. J Orthop Traumatol 2000;1:51-6.

23. Harris AM, Patterson BM, Sontich JK, Vallier HA. Results and outcomes after operative treatment of high-energy tibial plafond fractures. Foot Ankle Int 2006;27:256-65.

24. Koulouvaris P, Stafylas K, Mitsionis G, Vekris M, Mavrodontidis A, Xenakis T, et al. Long-term results of various therapy concepts in severe pilon fractures. Arch Orthop Trauma Surg 2007;127:313-20.

25. Bacon S, Smith WR, Morgan SJ, Hasenboehler E, Philips G, Williams A, et al. A retrospective analysis of comminuted intra-articular fractures of the tibial plafond: Open reduction and internal fixation versus external ilizarov fixation. Injury 2008;39:196-202.

26. Wang C, Li Y, Huang L, Wang M. Comparison of two-staged ORIF and limited internal fixation with external fixator for closed tibial plafond fractures. Arch Orthop Trauma Surg 2010;130:1289-97.

27. Davidovitch RI, Elkhechen RJ, Romo S, Walsh M, Egol KA. Open reduction with internal fixation versus limited internal fixation and external fixation for high grade pilon fractures (OTA type 43C). Foot Ankle Int 2011;32:955-61.

28. Richards JE, Magill M, Tressler MA, Shuler FD, Kregor PJ, Obremskey WT, et al. External fixation versus ORIF for distal intra-articular tibia fractures. Orthopedics 2012;35:e862-7.

29. Guo Y, Tong L, Li S, Liu Z. External fixation combined with limited internal fixation versus open reduction internal fixation for treating Ruedi-Allgower Type III pilon fractures. Med Sci Monit 2015;21:1662-7.

30. Cisneros LN, Gomez M, Alvarez C, Millan A, De Caso J, Soria L. Comparison of outcome of tibial plafond fractures managed by hybrid external fixation versus two-stage management with final plate fixation. Indian J Orthop 2016;50:123-30.

31. Wells GA, Shea B, O'Connell D, Petersen J, Welch V, Losos M, et al. The Newcastle-Ottawa Scale (NOS) for Assessing the Quality of Nonrandomized Studies in Meta-Analyses. Department of Epidemiology and Community Medicine, University of Ottawa, Canada; 2014. Available from: http://www.ohri.ca/programs/clinical_epidemiology/ oxford.asp. [Last accessed on 2017 Jul 15].

32. Ryan R, Hill S, Prictor M, McKenzie J; Cochrane Consumers and Communication Review Group. Study Quality Guide; 2013. Available from: http://cccrg.cochrane.org/authorresources. [Last accessed on 2017 Jul 15].

33. Endres T, Grass R, Biewener A, Barthel S, Zwipp H. Advantages of minimally-invasive reposition, retention, and ilizarov-(hybrid) fixation 
Younis, et al.

for pilon-tibial-fractures fractures with particular emphasis on $\mathrm{C} 2 / \mathrm{C} 3$ fractures. Unfallchirurg 2004;107:273-84.

34. Ketz J, Sanders R. Staged posterior tibial plating for the treatment of Orthopaedic Trauma Association 43C2 and 43C3 tibial pilon fractures. J Orthop Trauma 2012;26:341-7.

35. White TO, Guy P, Cooke CJ, Kennedy SA, Droll KP, Blachut PA, et al. The results of early primary open reduction and internal fixation for treatment of OTA 43.C-type tibial pilon fractures: A cohort study. J Orthop Trauma 2010;24:757-63.

36. Leonard M, Magill P, Khayyat G. Minimally-invasive treatment of high velocity intra-articular fractures of the distal tibia. Int Orthop 2009:33:1149-53.

37. Murphy CP, D'Ambrosia R, Dabezies EJ. The small pin circular fixator for distal tibial pilon fractures with soft tissue compromise. Orthopedics 1991;14:283-90

38. Tornetta P $3^{\text {rd }}$, Weiner L, Bergman M, Watnik N, Steuer J, Kelley M, et al. Pilon fractures: Treatment with combined internal and external fixation. J Orthop Trauma 1993;7:489-96.

39. Chaudhry H, Mundi R, Singh I, Einhorn TA, Bhandari M. How good is the orthopaedic literature? Indian J Orthop 2008;42:144-9.

40. Pollak AN, McCarthy ML, Bess RS, Agel J, Swiontkowski MF. Outcomes after treatment of high-energy tibial plafond fractures. J Bone Joint Surg Am 2003;85-A: 1893-900.

41. Boraiah S, Kemp TJ, Erwteman A, Lucas PA, Asprinio DE. Outcome following open reduction and internal fixation of open pilon fractures. J Bone Joint Surg Am 2010;92:346-52. 